\section{BIOPELÍCULAS Y PERSISTENCIA MICROBIANA EN LA INDUSTRIA ALIMENTARIA}

\author{
Paula Fernández-Gómez \\ Universidad de León \\ ORCID iD: https://orcid.org/0000-0001-9647-5251 \\ pafeg@unileon.es \\ Miguel Prieto \\ Universidad de León \\ ORCID iD: https://orcid.org/0000-0001-9202-3856 \\ miguel.prieto@unileon.es \\ Pablo S. Fernández-Escámez \\ Universidad Politécnica de Cartagena (ETSIA) \\ ORCID iD: https://orcid.org/0000-0002-4273-7268 \\ pablo.fernandez@upct.es \\ Mercedes López \\ Universidad de León \\ ORCID iD: https://orcid.org//0000-0003-2899-6391 \\ mmlopf@unileon.es \\ Avelino Alvarez-Ordóñez \\ Universidad de León \\ ORCID iD: http://orcid.org/0000-0002-9951-4786 \\ aalvo@unileon.es
}

Cómo citar este artículo/Citation: Fernández-Gómez, P., Prieto, M., Fernández-Escámez, P. S., López, M. y Alvarez-Ordóñez, A. (2020). Biopelículas y persistencia microbiana en la industria alimentaria. Arbor, 196 (795): a538. https://doi.org/10.3989/ arbor.2020.795n1002

Recibido: 26 febrero 2019. Aceptado: 29 octubre 2019.

RESUMEN: Este artículo de revisión examina la importancia que tienen las comunidades microbianas que colonizan los ambientes y equipos de procesado de alimentos formando biopelículas o biofilms en la persistencia microbiana en la industria alimentaria y consecuentemente, en la seguridad y la calidad de los alimentos. La atención se centra especialmente en biopelículas formadas por microorganismos no deseados, es decir, microorganismos alterantes y patógenos. Se presenta información sobre la variabilidad intraespecífica en la formación, la ecología y la arquitectura de las biopelículas, y los factores que influyen en su formación. Asimismo, se resume la información disponible sobre nuevos agentes o estrategias para el control de la formación o eliminación de biopelículas.

PALABRAS CLAVE: biofilms; persistencia; ecología microbiana; control; procesado de alimentos.

\section{BIOFILMS AND MICROBIAL PERSISTENCE IN THE FOOD INDUSTRY}

Copyright: (C) 2020 CSIC. Este es un artículo de acceso abierto distribuido bajo los términos de la licencia de uso y distribución Creative Commons Reconocimiento 4.0 Internacional (CC BY 4.0).

ABSTRACT: This review examines the importance that microbial communities colonizing food processing environments in the form of biofilms have on food safety and food quality. The focus is on biofilms of undesired microorganisms, i.e. pathogenic and spoilage microorganisms. Information is presented on intraspecies variability in biofilm formation, biofilm ecology and architecture and the factors influencing their formation. Finally, research on novel agents or strategies for the control of biofilm formation or its removal is summarized.

KEYWORDS: biofilms; persistence; microbial ecology; control; food processing. 


\section{INTRODUCCIÓN}

Un biofilm o biopelícula puede definirse como una comunidad microbiana caracterizada por su adhesión a una superficie sólida y por la producción de una matriz polimérica extracelular en la que están embebidos los microorganismos asociados. Esta matriz proporciona protección a las células microbianas y contribuye a la captación de nutrientes, así como a la adhesión a la superficie en cuestión. La formación de biopelículas es un comportamiento social generalmente coordinado a través de sistemas de comunicación célula - célula, o sistemas de "quorum sensing". Dichos sistemas de "quorum sensing" detectan fluctuaciones en la densidad celular a través del reconocimiento de pequeñas moléculas de señalización secretadas, llamadas autoinductores, y responden regulando la expresión de funciones celulares especializadas entre las que se encuentran las responsables de la adhesión inicial a superficies y el subsiguiente crecimiento y maduración de la biopelícula. De particular relevancia resulta el hecho de que las células microbianas dentro de una biopelícula son significativamente más resistentes a diferentes tipos de intervenciones antimicrobianas, dirigidas a controlar su aparición, y que las biopelículas pueden actuar como un reservorio de microorganismos persistentes. Se puede encontrar información más detallada sobre las propiedades ecológicas de las biopelículas y el comportamiento microbiano dentro de las mismas en Flemming et al. (2016) y Nadell, Drescher y Foster (2016).

En la industria alimentaria, las superficies y los equipos se encuentran frecuentemente colonizados por microorganismos en forma de biopelículas. En la mayoría de las ocasiones, esto representa un desafío y una preocupación, ya que las biopelículas formadas por microorganismos alterantes y patógenos pueden servir como fuente de contaminación cruzada de los alimentos, reduciendo así la efectividad de las estrategias de conservación de alimentos y comprometiendo la calidad y seguridad de los mismos (Coughlan, Cotter, Hill y Álvarez-Ordóñez, 2016). Por otro lado, las biopelículas formadas de manera controlada por microorganismos beneficiosos pueden representar una oportunidad, ya que pueden ser explotadas para aumentar el rendimiento y la calidad de las fermentaciones de alimentos o para desarrollar aplicaciones biotecnológicas centradas en mejorar la calidad y seguridad de los alimentos (Berlanga y Guerrero, 2016).

\section{LAS BIOPELÍCULAS COMO RESPONSABLES DE LA PERSIS- TENCIA MICROBIANA EN LA INDUSTRIA ALIMENTARIA}

Existe controversia acerca de si la persistencia microbiana en la industria alimentaria se debe a la presencia de nichos ambientales difíciles de limpiar y desinfectar, o a la colonización de los ambientes de procesado de alimentos por microorganismos que muestran capacidades especiales que les permiten sobrevivir en las condiciones adversas que imperan en las industrias alimentarias (Larsen et al., 2014). Entre otros factores, la capacidad de formar biopelículas se ha citado como un atributo que puede contribuir a la colonización de manera persistente de los ambientes de procesado de alimentos (Bridier et al., 2015). En este sentido, varios autores han tratado de evaluar si determinadas especies microbianas o genotipos recuperados de nichos industriales, o aislados persistentes comúnmente encontrados en industrias alimentarias, están mejor equipados para formar biopelículas en materiales de contacto con alimentos. Así, por ejemplo, un estudio de este tipo observó una mejor adhesión, tras 24 horas, entre 23 cepas persistentes de Listeria monocytogenes en relación con 73 cepas no persistentes (Wang, Ray, Hammons y Oliver, 2015), y Nowak y coautores llegaron a conclusiones similares, observando una formación de biopelículas significativamente mayor después de 48 horas a $30^{\circ} \mathrm{C}$ para aislamientos persistentes de L. monocytogenes $(n=8)$ recuperados de plantas de procesado de mejillones en relación con aislados no persistentes $(n=8)$ (Nowak et al., 2017).

En algunas ocasiones se han descrito diferencias estadísticamente significativas en la capacidad de formación de biopelículas entre cepas pertenecientes a diferentes serotipos o genotipos. Por ejemplo, se ha demostrado que cepas de L. monocytogenes pertenecientes a los serotipos $1 / 2$ b y $1 / 2 a$, es decir, aquellos que se aíslan con mayor frecuencia en ambientes de procesado de alimentos, forman biopelículas de manera más eficaz en medios altamente nutritivos a $20^{\circ} \mathrm{C}, 30^{\circ} \mathrm{C}$ y $37^{\circ} \mathrm{C}$ que cepas del serotipo $4 \mathrm{~b}$, es decir, aquel más frecuentemente vinculado a casos de infección humana (Kadam et al., 2013). Para Escherichia coli, se ha publicado que los aislados del seropatotipo A (0157: H7 y 0157: NM), más frecuentemente asociado a infecciones en humanos, poseen una mayor capacidad para formar biopelículas que los aislados de los seropatotipos B o C (Vogeleer, Tremblay, Jubelin, Jacques y Harel, 2016). 


\section{FACTORES QUE DETERMINAN LA FORMACIÓN DE BIOPELÍCULAS EN LA INDUSTRIA ALIMENTARIA}

En varias ocasiones se ha formulado la hipótesis de que los microorganismos que pueden activar funciones específicas, en particular la formación de biopelículas, en respuesta a algunos componentes de los alimentos o a señales ambientales presentes en el procesado de alimentos a nivel industrial, son también los más capaces de persistir en ambientes de procesado de alimentos, mediante la colonización de superficies y equipos. Teniendo esto en cuenta, varios autores han evaluado la formación de biopelículas por microorganismos de interés alimentario en medios suplementados con determinados componentes de los alimentos, o en diferentes condiciones ambientales que comúnmente prevalecen durante el procesado de los alimentos. A partir de tales estudios, es evidente que varios carbohidratos simples pueden modular la formación de biopelículas en bacterias. Los ejemplos incluyen la glucosa en Aeromonas hydrophila (Jahid, Lee, Kim y Ha, 2013), y la lactosa, que mejora la formación de biopelículas en S. aureus (Xue, Chen y Shang, 2014) y en Bacillus subtilis (Duanis-Assaf, Steinberg, Chai y Shemesh, 2016). Otros constituyentes de los alimentos que se ha demostrado que aumentan la formación de biopelículas son la L-leucina en L. monocytogenes (Skovager et al., 2013) y el ácido butírico, liberado durante la lipólisis de la leche, en Bacillus spp. (Pasvolsky, Zakin, Ostrova y Shemesh, 2014). Además, también se ha observado que la formación de biopelículas de Streptococcus thermophilus en acero inoxidable depende de la presencia de proteínas de la leche (Bassi, Cappa, Gazzola, Orrù y Cocconcelli, 2017).

La disponibilidad de determinados minerales es otro factor que puede influir en la formación de biopelículas bacterianas. En Bacillus cereus se ha demostrado que el acero inoxidable representa un material de contacto más favorable para la formación y maduración de biopelículas que el poliestireno, y este efecto se relacionó con una mayor disponibilidad de hierro (Hayrapetyan, Muller, Tempelaars, Abee y Nierop Groot, 2015).

Varios autores han evaluado la capacidad de diferentes microorganismos para formar biopelículas en presencia de extractos de alimentos. En el caso de Campylobacter jejuni y Campylobacter coli, la formación de biopelículas sobre vidrio, poliestireno y acero inoxidable fue mayor cuando el medio de crecimiento se suplementaba con un extracto de carne de pollo, que era una fuente adicional de nutrientes y cubría y acondicionaba las superficies abióticas (Brown et al., 2014). También se obtuvieron resultados similares para Salmonella spp. y Campylobacter spp. en poliestireno y superficies de vidrio utilizando extractos de carne de cerdo y de pollo (Li et al., 2017), y para Salmonella spp. en distintos materiales de contacto con los alimentos utilizando extracto de pescado (Dhowlaghar et al., 2018).

Varios grupos de investigación han estudiado y modelizado cómo distintas condiciones medioambientales que prevalecen durante el procesado de alimentos influyen en la formación de biopelículas, en aras de obtener información útil para la prevención o control de los mismos (Dimakopoulou-Papazoglou, Lianou y Koutsoumanis, 2016; Iliadis, Daskalopoulou, Simões y Giaouris, 2018).

Numerosos autores han reconocido el papel que juegan las biopelículas como reservorio de microorganismos resistentes a distintos agentes antimicrobianos y condiciones de estrés. Además, las biopelículas también pueden servir como fuente de formas celulares de resistencia. De hecho, se ha demostrado que las bacterias formadoras de esporas, como Bacillus spp., son capaces de esporular dentro de las biopelículas liberando estas esporas altamente resistentes al entorno circundante, lo que aumenta el riesgo de contaminación cruzada de los alimentos (Faille et al., 2014). Además, también se han detectado células en un estado viable pero no cultivable en biopelículas formadas por L. monocytogenes, especialmente después de la aplicación de tratamientos de limpieza y desinfección (Gião y Keevil, 2014; Overney et al., 2017).

Finalmente, también se ha identificado una interconexión entre las respuestas de adaptación al estrés y la formación de biopelículas, que podría ser la responsable de la mayor robustez de las células que conforman las biopelículas. Así, por ejemplo, el regulador de la respuesta general al estrés en bacterias Gram negativas, el factor alternativo Rpos, ha sido identificado como un factor clave para el establecimiento de biopelículas maduras en E. coli (Álvarez-Ordóñez et al., 2013; Chen et al., 2013) y también se ha observado un vínculo entre el potencial de formación de biopelículas de E. coli y su termorresistencia (Marti et al., 2017).

\section{ECOLOGÍA Y ARQUITECTURA DE LAS BIOPELÍCULAS MICROBIANAS}

En general, se sabe que dentro de una biopelícula coexisten bacterias de múltiples especies formando consorcios complejos, donde las relaciones de cooperativismo y competencia son comunes y contri- 
buyen a dar forma a la estructura de la población, condicionando su funcionalidad (Giaouris et al., 2015). Las interacciones de las principales bacterias patógenas transmitidas por los alimentos con otras bacterias relacionadas con los alimentos o con los miembros de la microbiota residente que colonizan los ambientes de procesado de alimentos se han estudiado detalladamente en los últimos años en ensayos in vitro. En algunos casos, se han observado interacciones sinérgicas, en las cuales determinadas cepas de patógenos bacterianos transmitidos por los alimentos que son malos formadores de biopelículas aprovechan su interacción con otras cepas productoras de biopelículas fuertes para colonizar los materiales en contacto con los alimentos. Así, por ejemplo, se ha demostrado que L. monocytogenes interactúa de forma sinérgica con algunas cepas de Enterococcus faecalis y Enterococcus faecium (da Silva Fernandes, Kabuki y Kuaye, 2015). Además, alrededor del $20 \%$ de una amplia gama de cócteles multiespecíficos, preparados con cepas originalmente aisladas de dos sitios de muestreo en una planta de procesado de productos cárnicos, mostraron una mayor formación de biopelículas en comparación con las biopelículas monoespecíficas formadas individualmente por las distintas cepas estudiadas ( $R \varnothing$ der et al., 2015). Aunque en la mayoría de los casos todavía no se conoce la causa de estos comportamientos sinérgicos, se ha propuesto que la coagregación, el reconocimiento específico y co-adherencia y la alimentación cruzada entre cepas pueden ser mecanismos involucrados en estas interacciones cooperativas (Stevens et al., 2015; Herschend et al., 2017). Por otro lado, varios autores han descrito la existencia de interacciones competitivas, donde un miembro del consorcio supera o elimina a otros miembros de la comunidad y se convierte en dominante. Esto se ha demostrado para distintas especies de bacterias Gram negativas, que han mostrado capacidad para competir frente a L. monocytogenes, dominando las biopelículas multi-especie formadas (Daneshvar Alavi y Truelstrup Hansen, 2013; Rodríguez-López, Saá-Ibusquiza, Mosquera-Fernández y López-Cabo, 2015; Heir, Møretrø, Simensen y Langsrud, 2018; Papaioannou, Giaouris, Berillis y Boziaris, 2018). Otros comportamientos competitivos similares, que dan como resultado el desplazamiento de cepas de otras bacterias patógenas transmitidas por los alimentos, como E. coli, Bacillus spp. y S. aureus, también se han descrito en la literatura (Wang et al., 2015; Rosenberg et al., 2016; Makovcova et al., 2017, Visvalingam, Ells y Yang, 2017).
La mayoría de los datos publicados en la literatura sobre la formación de biopelículas in vitro por bacterias asociadas a los alimentos se basan en simples ensayos cuantitativos de tinción, que no proporcionan información sobre la estructura microscópica y arquitectura de la biopelícula. Sin embargo, cada vez es más evidente que existe una micro-heterogeneidad dentro de las biopelículas (Liu et al., 2015; Gingichashvili et al., 2017; Tack, Nimmegeers, Akkermans, Hashem y van Impe, 2017), con la existencia de fenómenos de diferenciación metabólica entre las distintas células que las constituyen. También se sabe que varios factores relacionados con los alimentos, como la concentración de nutrientes, la disponibilidad de oxígeno, la composición de la matriz alimentaria, la concentración de azúcar y las condiciones hidrodinámicas, pueden influir en la arquitectura de la biopelícula (Cherifi, Jacques, Quessy y Fravalo, 2017; Tarifa, Genovese, Lozano y Brugnoni, 2018; Turonova et al., 2015).

\section{CONTROL DE BIOPELÍCULAS MICROBIANAS EN LA IN- DUSTRIA ALIMENTARIA}

Teniendo en cuenta el papel de las biopelículas como un reservorio de microorganismos potencialmente problemáticos, que luego pueden contaminar los alimentos y causar su deterioro o su implicación en casos de toxi-infección alimentaria, se ha dedicado gran esfuerzo investigador a mejorar los métodos y estrategias disponibles para eliminarlos de ambientes industriales o desarrollar nuevas herramientas de inhibición o de eliminación que sean más efectivas, económicas y sostenibles.

Las industrias alimentarias basan sus protocolos de limpieza y desinfección en el uso de desinfectantes y biocidas que permitan establecer barreras a la entrada de microorganismos no deseados controlando la colonización de superficies y equipos en contacto con alimentos. Los biocidas se emplean generalmente en concentraciones muy por encima de sus concentraciones mínimas inhibitorias para todos los microorganismos diana principales y, por lo tanto, deberían poder garantizar la inactivación microbiana, evitando así la supervivencia de microorganismos peligrosos. Sin embargo, es bien sabido que los biocidas y otros antimicrobianos son menos efectivos en la inactivación de células en estado sésil (formando biopelículas) que en estado planctónico. De hecho, varias publicaciones, que evalúan la tolerancia de las principales bacterias patógenas transmitidas por los alimentos a una amplia gama de desinfectantes de uso industrial o sus compuestos activos a sus concentraciones de uso 
industrial, han demostrado que estos no son capaces de inactivar completamente los microorganismos diana formando biopelículas (Chaitiemwong, Hazeleger y Beumer, 2014; Chylkova, Cadena, Ferreiro y Pitesky, 2017; Fagerlund, Langsrud, Heir, Mikkelsen y Møretr $\varnothing$, 2016; Martin et al., 2016).

Además, varios estudios han descrito que la tolerancia a diferentes biocidas es mayor en las biopelículas mixtas o multi-especie que en las biopelículas formadas por una única especie (Bridier et al., 2015; Giaouris, Chorianopoulos, Doulgeraki y Nychas, 2013; Wang, Kalchayanand, Schmidt y Harhay, 2013), y que la composición de la matriz de la biopelícula y las características de la superficie influyen en gran medida en la efectividad del biocida (Bas, Kramer y Stopar, 2017; Fagerlund et al., 2016). También es importante tener en cuenta que los microorganismos que colonizan las plantas de procesado de alimentos se ven frecuentemente expuestos a concentraciones subinhibitorias de biocidas, en nichos particulares (por ejemplo, en grietas y otros sitios de difícil acceso) o como consecuencia de su uso inadecuado, como, por ejemplo, debido a una formulación errónea, almacenamiento inadecuado o aplicación en superficies húmedas, con la consiguiente dilución del compuesto a concentraciones que pueden ser subletales. Es importante destacar que varios autores han descrito que la adaptación previa a algunos biocidas y compuestos activos, como el nitrito de sodio y el hipoclorito de sodio en $E$. coli, el cloruro de benzalconio en $L$. monocytogenes, el hipoclorito de sodio en $S$. aureus y S. Typhimurium, el etanol y la cloramina $T$ en $S$. aureus, y el fosfato trisódico, ácido acético, hipoclorito de sodio y dos desinfectantes comerciales en $C$. jejuni, puede favorecer la formación de biopelículas (Buzón-Durán, Alonso-Calleja, Riesco-Peláez y Capita, 2017; Capita, Buzón-Durán, Riesco-Peláez y Alonso-Calleja, 2017; Ortiz, López y Martínez Suárez, 2014; Slany, Oppelt y Cincarova, 2017; Techaruvichit et al., 2016).

La modificación de los materiales utilizados en la industria alimentaria se ha revelado como un medio prometedor para prevenir la formación de biopelículas. Dado que la formación de una biopelícula implica como primer paso la unión o adhesión de células planctónicas a una superficie sólida, si dicha superficie se modifica en cierta medida, por ejemplo, alterando su morfología o propiedades físico-químicas (hidrofobicidad, hidrofilicidad, carga eléctrica, etc.), la adhesión microbiana y, en consecuencia, el crecimiento y la maduración de la biopelícula pueden ser controlados. La adherencia bacteriana a las superficies industriales se puede minimizar utilizando topografías controladas, como demostraron Hsu et al. (2013) empleando superficies de sílice y alúmina. Los resultados obtenidos por estos autores evidenciaron que las células de E. coli, Listeria innocua y P. fluorescens cambiaban su morfología, incluyendo el número y tamaño de los apéndices celulares, dependiendo de la topografía a nanoescala del material de superficie. De hecho, también se ha demostrado que las topografías a nanoescala de poros pequeños inhiben la unión dependiente de flagelos de $E$. coli a las superficies de alúmina (Feng et al., 2014).

Dado el importante efecto que la topografía y las propiedades físico-químicas de la superficie tienen en las etapas iniciales de la formación de biopelículas, varias iniciativas se han centrado recientemente en desarrollar recubrimientos que modifiquen dichas propiedades de superficie, reduciendo así la adhesión bacteriana y mejorando la efectividad de los métodos de limpieza y desinfección. De este modo, se han desarrollado recubrimientos antiincrustantes efectivos en acero inoxidable usando distintos precursores u órgano-polímeros (Gkana, Doulgeraki, Chorianopoulos y Nychas, 2017; Gomes, Deschamps, Briandet y Mergulhão, 2018; Huang, Chen, Nugen y Goddard, 2016). Incluso se ha demostrado la efectividad de estos recubrimientos en entornos reales utilizando intercambiadores de calor de placas con superficie modificada durante una sesión de pasteurización de leche de 17 horas (Jindal, Anand, Metzger y Amamcharla, 2018). Aunque la mayoría de los estudios que prueban el potencial de los recubrimientos antiincrustantes se han centrado en las superficies de acero inoxidable, se han evaluado otros materiales, como el polietileno de baja densidad (Hüwe et al., 2018). Además, en otras ocasiones, se han desarrollado recubrimientos superficiales que incorporan compuestos antimicrobianos que también han demostrado capacidad para prevenir la formación de biopelículas por varios patógenos transmitidos por los alimentos (Cossu, Si, Sun y Nitin, 2017; Fialho et al., 2018; Kim et al., 2017).

El desarrollo de nuevos agentes desinfectantes más efectivos, capaces de eliminar las biopelículas bacterianas de las superficies y equipos industriales, es un área de investigación prioritaria. Debido a su capacidad para degradar las sustancias poliméricas que conforman la matriz extracelular de las biopelículas, los detergentes enzimáticos se consideran agentes innovadores respetuosos con el medio ambiente y útiles para facilitar la eliminación de biopelículas. Como el ADN extracelular 
es un componente habitual de la matriz de biopelículas microbianas, las enzimas con acción DNasa, solas o combinadas con otras estrategias de saneamiento, pueden facilitar la eliminación de las biopelículas, como se ha demostrado recientemente para $C$. jejuni (Brown, Hanman, Reuter, Betts y van Vliet, 2015) o $L$. monocytogenes (Nguyen y Burrows, 2014). Además, las proteasas, como la proteinasa K (Nguyen y Burrows, 2014), las lipasas (Kiran, Lipton, Kennedy, Dobson y Selvin, 2014) o las enzimas que degradan carbohidratos, como la $\beta$-glucanasa y la $\alpha$-amilasa (Araújo et al., 2017), por su actividad lítica sobre otros componentes de la matriz extracelular de las biopelículas, también se han propuesto como posibles candidatos para ser utilizados como herramientas de control.

El potencial del agua electrolizada, producida a través de la electrólisis de una solución acuosa de cloruro de sodio, como agente de limpieza y desinfección ha sido demostrado en varias ocasiones. De hecho, se ha descrito que el agua electrolizada acidificada o ligeramente acidificada elimina eficazmente biopelículas de L. innocua, L. monocytogenes, Vibrio parahaemolyticus, E. coli y B. cereus (Han et al., 2017; Hussain, Kwon, Tango y Oh, 2018; Jeon, Kwon y Yoon, 2018). Curiosamente, el agua neutra electrolizada también posee actividad anti-biopelícula (Moradi y Tajik, 2017) y se ha demostrado recientemente que el agua electrolizada básica tiene una mayor capacidad de dispersión de biopelículas de $B$. cereus que el agua electrolizada acidificada o ligeramente acidificada, aunque mostró una menor actividad bactericida contra células planctónicas (Hussain et al., 2018).

Las actividades de investigación también se están centrando en la identificación de nuevos compuestos antimicrobianos que puedan ser incluidos en nuevas formulaciones para su uso como desinfectantes sostenibles. En particular, en los últimos años, una amplia gama de estudios han evaluado la efectividad de distintos compuestos de origen natural, incluidos varios aceites esenciales o extractos obtenidos de plantas, alimentos $u$ otros productos derivados, para la inhibición de la formación de biopelículas o la eliminación de biopelículas ya existentes. Algunos de estos nuevos compuestos y extractos ejercen un efecto bactericida directo sobre los microorganismos, mientras que otros muestran actividades indirectas de inhibición de biopelículas, relacionadas principalmente con la inhibición de sistemas de "quorum sensing" (Coughlan et al., 2016). El lector puede encontrar más información relacionada con este campo de investigación en Ashraf et al. (2014).
También se han propuesto algunas nuevas tecnologías de inactivación microbiana como herramientas alternativas para el control de biopelículas en la industria alimentaria. Entre ellas, los plasmas atmosféricos no térmicos han recibido una gran atención, ya que han demostrado una alta capacidad desinfectante contra biopelículas de un amplio espectro de microorganismos (Puligundla y Mok, 2017). De hecho, los plasmas no térmicos han sido capaces de eliminar con éxito biopelículas formadas por Salmonella en vidrio (Niemira, Boyd y Sites, 2014), E. coli, L. monocytogenes y $S$. aureus en tereftalato de polietileno (Ziuzina, Boehm, Patil, Cullen y Bourke, 2015) y P. aeruginosa, Pseudomonas libanensis, Enterobacter cloacae, Kocuria carniphila, Staphylococcus epidermidis y B. subtilis en acero inoxidable (Mai-Prochnow, Clauson, Hong y Murphy, 2016). Sin embargo, se debe prestar atención a los subproductos potencialmente tóxicos que pueden generar esas tecnologías en soluciones acuosas ricas en materia orgánica. Además, otras tecnologías de descontaminación física de superficies, que se han desarrollado o investigado en los últimos años para la inactivación de microorganismos en biopelículas son los tratamientos fotodinámicos con luz a 405 nm (McKenzie et al., 2013) o luz ultravioleta pulsada (Montgomery y Banerjee, 2015), la ozonización de superficies (Nicholas, Dunton, Tatham y Fielding, 2013), o el tratamiento de superficies con ultrasonidos (Axelson et al., 2013) o dióxido de cloro gaseoso (Nam et al., 2014).

El uso de microorganismos vivos o sus metabolitos para la exclusión competitiva o la inactivación de microorganismos alterantes o patógenos en biopelículas es un campo que está recibiendo creciente atención. Dentro de este campo, se está investigando la actividad anti-biopelícula de varias bacterias ácido-lácticas, principalmente productoras de bacteriocinas, y de diversos bacteriófagos. Varios estudios han demostrado la capacidad de las bacteriocinas nisina, subtilomicina, lichenicidina, enterocina B3A-B3B, enterocina AS-48 y sonorensina, para inhibir la formación de biopelículas o eliminar biopelículas formadas por diferentes bacterias patógenas (Al-Seraih et al., 2017; Bolocan et al., 2017; Caballero Gómez, Abriouel, Grande, Pérez Pulido y Gálvez, 2013; Chopra, Singh, Kumar Jena y Sahoo, 2015; Field, O'Connor, Cotter, Ross y Hill, 2016). Además, otros metabolitos bacterianos, como algunos surfactantes (Coronel-León, Marqués, Bastida y Manresa, 2016), endoglicosidasas (Yu et al., 2015) y ácidos grasos insaturados (Sepehr, Rahmani-Badi, Babaie-Naiej y Soudi, 2014) se han aplicado con éxito para evitar la formación de biopelículas, y algunos microorganismos incluso han demostrado capacidad para inhibir los sis- 
temas de "quorum sensing" de otros microorganismos competidores (Coughlan et al., 2016). No obstante, en lugar de purificar y usar estos metabolitos secundarios como moléculas inhibidoras de biopelículas, varios autores han evaluado la utilización directa de aquellos microorganismos inocuos que los producen como una estrategia de control de biopelículas en la industria alimentaria (Kim, Bang, Kim, Beuchat y Ryu, 2013; Son, Park, Beuchat, Kim y Ryu, 2016). En este sentido, un ensayo de exclusión competitiva demostró que la colonización de los desagües de una planta de procesado de carne de pollo por cepas de L. lactis y Enterococcus durans reducía la persistencia de L. monocytogenes en los mismos (Zhao et al., 2013). Asimismo, se ha demostrado que las biopelículas naturales presentes en los estantes de madera utilizados en la maduración del queso francés "Reblochon de Savoie" previenen el crecimiento de L. monocytogenes (Mariani et al., 2011), y que el desarrollo de biopelículas de L. lactis subsp. cremoris en las cubas de madera utilizadas para la producción del queso DOP Vastedda della valle del Belice permite la reducción de la diversidad microbiana y estabiliza los atributos sensoriales de los quesos producidos (Gaglio et al., 2016).

Debido a su elevada especificidad, los bacteriófagos han sido reconocidos como herramientas adecuadas para eliminar biopelículas formadas por un determinado microorganismo alterante o patógeno (Gutiérrez, Rodríguez-Rubio, Martínez, Rodríguez y García, 2016). Así, varios estudios han pretendido en la última década identificar nuevos bacteriófagos efectivos en la eliminación de biopelículas de los principales patógenos transmitidos por los alimentos, y, de hecho, algunos fagos han sido postulados como agentes de control biológico contra las biopelículas de Cronobacter sakazakii, E. coli, S. aureus, Salmonella spp. y L. monocytogenes (Chaitiemwong et al., 2014; Endersen et al., 2017; González et al., 2017; Sadekuzzaman, Yang, Mizan y Ha, 2017; Shafique, Alvi, Abbas y ur Rehman, 2017). Además, algunas enzimas líticas derivadas de fagos, como las endolisinas, también han demostrado actividad contra las biopelículas bacterianas (Gutiérrez, Ruas-Madiedo, Martínez, Rodríguez y García, 2014).

\section{CONCLUSIONES}

Las biopelículas representan una fuente de contaminación cruzada de alimentos por microorganismos alterantes y patógenos, y por ello han recibido una gran atención, con actividades de investigación centradas principalmente en la comprensión de los factores bióticos y abióticos que influyen en la formación y maduración de las biopelículas, y en la identificación, desarrollo y validación de estrategias novedosas para su control. Sin embargo, la mayoría de estas actividades de investigación se basan en modelos de biopelículas in vitro, normalmente en monoespecie o en modelos duales, que utilizan cepas domesticadas de dos especies diferentes, habitualmente pertenecientes a los grupos patógenos de transmisión alimentaria más relevantes. Sin embargo, poco se sabe acerca de la ecología y estructura de biopelículas formadas en entornos reales, en superficies y equipos de trabajo en plantas de procesado de alimentos. El desarrollo de herramientas novedosas para la evaluación de comunidades microbianas complejas, basadas principalmente en el análisis por secuenciación masiva de muestras de ADN obtenidas de nichos ambientales concretos, puede revolucionar el estudio de los biofilms en la industria alimentaria, ya que permitirá la caracterización in situ de biopelículas silvestres en las propias instalaciones de procesado de alimentos. En este sentido, la introducción temprana de medidas de control se verá facilitada por la disponibilidad de prototipos de secuenciadores miniaturizados, con potencial para ser utilizados in situ, generando resultados en tiempo real (Benítez-Páez y Sanz, 2017) que, en combinación con las mejoras en las metodologías disponibles para la recuperación completa de las células asociadas a biopelículas en planes de muestreo de superficies y equipos, y con el desarrollo de nuevas bases de datos de genes relacionados con la formación de biopelículas y la persistencia microbiana, permitirán el diagnóstico y la caracterización en tiempo real de comunidades microbianas asociadas a biopelículas silvestres.

Uno de los principales desafíos existentes que la comunidad científica necesita abordar es el desarrollo de nuevas herramientas capaces de prevenir la formación de biopelículas o eliminar las existentes de una manera efectiva, evitando la aparición de resistencias. Las actividades en este sentido se centran actualmente en múltiples frentes, desde la identificación o el descubrimiento de nuevos antimicrobianos para ser incluidos en las nuevas formulaciones de biocidas, hasta el diseño de estrategias de descontaminación física efectivas en la inactivación de células asociadas con biopelículas en materiales en contacto con alimentos o el desarrollo de nuevos agentes de biocontrol que explotan las interacciones microbianas para atacar específicamente las biopelículas formadas por microorganismos peligrosos, sin presentar efectos antimicrobianos sobre las biopelículas formadas por microorganismos potencialmente beneficiosos. No obstante, es de prever que no será posible encontrar 
una "bala de plata" y que se necesitarán enfoques combinados, donde los agentes de control recientemente desarrollados se utilicen de manera inteligente en sinergia con metodologías de desinfección convencionales, para garantizar la eliminación de aquellos microorganismos peligrosos que colonizan de manera persistente los ambientes de procesado de alimentos.

\section{AGRADECIMIENTOS}

Los autores agradecen la financiación del Ministerio de Ciencia, Innovación y Universidades (AGL201678085-P y AGL2017-82779-C2-2-R). Paula FernándezGómez es becaria pre-doctoral de la Junta de Castilla y León (BOCYL-D-15122017-4).

\section{BIBLIOGRAFÍA}

Al-Seraih, A., Belguesmia, Y., Baah, J., Szunerits, S., Boukherroub, R. y Drider, D. (2017). Enterocin B3A-B3B produced by $L A B$ collected from infant faeces: potential utilization in the food industry for Listeria monocytogenes biofilm management. Antonie van Leeuwenhoek. International Journal of General and Molecular Microbiology, 110 (2), pp. 205-219. https://doi.org/10.1007/ s10482-016-0791-5

Álvarez-Ordóñez, A., Alvseike, O., Omer, M. K., Heir, E., Axelsson, L., Holck, A. y Prieto, M. (2013). Heterogeneity in resistance to food-related stresses and biofilm formation ability among verocytotoxigenic Escherichia coli strains. International Journal of Food Microbiology, 161 (3), pp. 220-230. https://doi.org/10.1016/j.ijfoodmicro.2012.12.008

Araújo, P. A., Machado, I., Meireles, A., Leiknes, T. O., Mergulhão, F., Melo, L. F. y Simões, M. (2017). Combination of selected enzymes with cetyltrimethylammonium bromide in biofilm inactivation, removal and regrowth. Food Research International, 95, pp. 101-107. https:// doi.org/10.1016/j.foodres.2017.02.016

Ashraf, M. A., Ullah, S., Ahmad, I., Qureshi, A. K., Balkhair, K. S. y Abdur Rehman, M. (2014). Green biocides, a promising technology: Current and future applications to industry and industrial processes. Journal of the Science of Food and Agriculture, 94 (3), pp. 388-403. https:// doi.org/10.1002/jsfa.6371

Axelson, L., Holck, A., Rud, I., Samah, D., Tierce, P., Favre, M. y Kure, C. F. (2013). Cleaning of conveyor belt materials using ultrasound in a thin layer of water. Journal of Food Protection, 76 (8), pp. 1401-1407. https://doi. org/10.4315/0362-028X.JFP-12-563

Bas, S., Kramer, M. y Stopar, D. (2017). Biofilm surface density determines biocide effectiveness. Frontiers in Microbiology, 8, 2443. https://doi.org/10.3389/ fmicb.2017.02443

Bassi, D., Cappa, F., Gazzola, S., Orrù, L. y Cocconcelli, P. S. (2017). Biofilm formation on stainless steel by Streptococcus thermophilus UC8547 in milk environments is mediated by the proteinase PrtS. Applied and Environmental Microbiology, 83 (8), e02840-16. https://doi. org/10.1128/AEM.02840-16

Benítez-Páez, A. y Sanz, Y. (2017). Multilocus and long amplicon sequencing approach to study microbial diversity at species level using the MinION ${ }^{\mathrm{TM}}$ portable nanopore sequencer. GigaScience, 6 (7), pp. 1-12. https://doi.org/10.1093/ gigascience/gix043

Berlanga, M. y Guerrero, R. (2016). Living together in biofilms: The microbial cell factory and its biotechnological implications. Microbial Cell Factories, 15, 165. https://doi.org/10.1186/s12934-0160569-5

Bolocan, A. S., Pennone, V., O'Connor, P. M., Coffey, A., Nicolau, A. I., McAuliffe, O. y Jordan, K. (2017). Inhibition of Listeria monocytogenes biofilms by bacteriocin-producing bacteria isolated from mushroom substrate. Journal of Applied Microbiology, 122 (1), pp. 279-293. https://doi.org/10.1111/jam.13337

Bridier, A., Sanchez-Vizuete, P., Guilbaud, M., Piard, J. C., Naïtali, M. y Briandet, R. (2015). Biofilm-associated persistence of food-borne pathogens. Food Microbiology, 45 (Pt B), pp. 167-178. https:// doi.org/10.1016/j.fm.2014.04.015

Brown, H. L., Hanman, K., Reuter, M., Betts, R. P. y Vliet, A. H. M. van (2015). Campylobacter jejuni biofilms contain extracellular DNA and are sensitive to DNase I treatment. Frontiers in Microbiology, 6, 699. https://doi.org/10.3389/ fmicb.2015.00699
Brown, H. L., Reuter, M., Salt, L. J., Cross, K. L., Betts, R. P. y Vliet, A. H. M. (2014). Chicken juice enhances surface attachment and biofilm formation of Campylobacter jejuni. Applied and Environmental Microbiology, 80 (22), pp. 7053-7060. https://doi.org/10.1128/AEM.02614-14

Buzón-Durán, L., Alonso-Calleja, C., RiescoPeláez, F. y Capita, R. (2017). Effect of subinhibitory concentrations of biocides on the architecture and viability of MRSA biofilms. Food Microbiology, 65, pp. 294-301. https://doi.org/10.1016/j.fm.2017.01.003

Caballero Gómez, N., Abriouel, H., Grande, M. J., Pérez Pulido, R. y Gálvez, A. (2013). Combined treatments of enterocin AS-48 with biocides to improve the inactivation of methicillin-sensitive and methicillin-resistant Staphylococcus aureus planktonic and sessile cells. International Journal of Food Microbiology, 163 (2-3), pp. 96-100. https://doi. org/10.1016/j.ijfoodmicro.2013.02.018

Capita, R., Buzón-Durán, L., Riesco-Peláez, F. y Alonso-Calleja, C. (2017). Effect of sub-lethal concentrations of biocides on the structural parameters and viability of the biofilms formed by Salmonella Typhimurium. Foodborne Pathogens and Disease, 14 (6), pp. 350-356. https://doi.org/10.1089/fpd.2016.2241

Chaitiemwong, N., Hazeleger, W. C. y Beumer, R. R. (2014). Inactivation of Listeria monocytogenes by disinfectants and bacteriophages in suspension and stainless steel carrier tests. Journal of Food Protection, 77 (12), pp. 2012-2020. https://doi. org/10.4315/0362-028X.JFP-14-151

Chen, C. Y., Hofmann, C. S., Cottrell, B. J., Strobaugh, T. P., Paoli, G. C., Nguyen, L. H., Yan, X. y Uhlich, G. A. (2013). Phenotypic and genotypic characterization of biofilm forming capabilities in non-0157 Shiga toxin-producing Escherichia coli strains. PLOS ONE, 8 (12), e84863. https://doi. org/10.1371/journal.pone.0084863 
Cherifi, T., Jacques, M., Quessy, S. y Fravalo, P. (2017). Impact of nutrient restriction on the structure of Listeria monocytogenes biofilm grown in a microfluidic system. Frontiers in Microbiology 8, 864. https://doi.org/10.3389/ fmicb.2017.00864

Chopra, L., Singh, G., Kumar Jena, K. y Sahoo, D. K. (2015). Sonorensin: A new bacteriocin with potential of an antibiofilm agent and a food biopreservative. Scientific Reports, 5, 13412. https://doi.org/10.1038/srep13412

Chylkova, T., Cadena, M., Ferreiro, A. y Pitesky, M. (2017). Susceptibility of Salmonella biofilm and planktonic bacteria to common disinfectant agents used in poultry processing. Journal of Food Protection, 80 (7), pp. 1072-1079. https:// doi.org/10.4315/0362-028X.JFP-16-393

Coronel-León, J., Marqués, A. M., Bastida, J. y Manresa, A. (2016). Optimizing the production of the biosurfactant lichenysin and its application in biofilm control. Journal of Applied Microbiology, 120 (1), pp. 99-111. https://doi. org/10.1111/jam.12992

Cossu, A., Si, Y., Sun, G. y Nitin, N. (2017). Antibiofilm effect of poly(vinyl alcoholcoethylene) halamine film against Listeria innocua and Escherichia coli 0157:H7. Applied and Environmental Microbiology, 83 (19), e00975-17. https://doi.org/10.1128/AEM.00975-17

Coughlan, L. M., Cotter, P. D., Hill, C. y Alvarez-Ordóñez, A. (2016). New weapons to fight old enemies: Novel strategies for the (bio)control of bacterial biofilms in the food industry. Frontiers in Microbiology, 7, 1641. https://doi. org/10.3389/fmicb.2016.01641

Daneshvar Alavi, H. E. y Truelstrup Hansen, L. (2013). Kinetics of biofilm formation and desiccation survival of Listeria monocytogenes in single and dual species biofilms with Pseudomonas fluorescens, Serratia proteamaculans or Shewanella baltica on food-grade stainless steel surfaces. Biofouling, 29 (10), pp. 1253-1268. https://doi.org/10.1080/08 927014.2013.835805

Dhowlaghar, N., De Abrew Abeysundara, P., Nannapaneni, R., Schilling, M. W., Chang, S., Cheng, W. H. y Sharma, C. S. (2018). Biofilm formation by Salmonella spp. in catfish mucus extract under industrial conditions. Food Microbiology, 70, pp. 172-180. https://doi. org/10.1016/j.fm.2017.09.016
Dimakopoulou-Papazoglou, D., Lianou, A. y Koutsoumanis, K. P. (2016). Modelling biofilm formation of Salmonella enterica ser. Newport as a function of $\mathrm{pH}$ and water activity. Food Microbiology, 53 (Pt B), pp. 76-81. https://doi. org/10.1016/j.fm.2015.09.002

Duanis-Assaf, D., Steinberg, D., Chai, Y. y Shemesh, M. (2016). The LuxS based quorum sensing governs lactose induced biofilm formation by Bacillus subtilis. Frontiers in Microbiology, 6, 1517. https://doi.org/10.3389/ fmicb.2015.01517

Endersen, L., Buttimer, C., Nevin, E., Coffey, A., Neve, H., Oliveira, H., Lavigne, R. y O'Mahony, J. (2017). Investigating the biocontrol and anti-biofilm potential of a three phage cocktail against Cronobacter sakazakii in different brands of infant formula. International Journal of Food Microbiology, 253, pp. 1-11. https://doi. org/10.1016/j.ijfoodmicro.2017.04.009

Fagerlund, A., Langsrud, S., Heir, E., Mikkelsen, M. I. y Møretrø, T. (2016). Biofilm matrix composition affects the susceptibility of food associated staphylococci to cleaning and disinfection agents. Frontiers in Microbiology, 7, 856. https://doi.org/10.3389/ fmicb.2016.00856

Faille, C., Bénézech, T., Midelet-Bourdin, G., Lequette, Y., Clarisse, M., Ronse, G., Ronse, A. y Slomianny, C. (2014). Sporulation of Bacillus spp. within biofilms: A potential source of contamination in food processing environments. Food Microbiology, 40, pp. 64-74. https:// doi.org/10.1016/j.fm.2013.12.004

Feng, G., Cheng, Y., Wang, S. Y., Hsu, L. C., Feliz, Y., Borca-Tasciuc, D. A., Worobo, R. W. y Moraru, C. I. (2014). Alumina surfaces with nanoscale topography reduce attachment and biofilm formation by Escherichia coli and Listeria spp. Biofouling, 30 (10), pp. 1253-1268. https://doi.org/ 10.1080/08927014.2014.976561

Fialho, J. F. Q., Naves, E. A. A., Bernardes, P. C., Ferreira, D. C., Anjos, L. D. dos, Gelamo, R. V., Sá, J. P. N. de y Andrade, N. J. de (2018). Stainless steel and polyethylene surfaces functionalized with silver nanoparticles. Food Science and Technology International, 24 (1), pp. 87-94. https://doi. org/10.1177/1082013217731414

Field, D., O'Connor, R., Cotter, P. D., Ross, R. P. y Hill, C. (2016). In vitro activities of nisin and nisin derivatives alone and in combination with antibiotics against Staphylococcus biofilms. Frontiers in Microbiology, 7, 508. https://doi. org/10.3389/fmicb.2016.00508

Flemming, H. C., Wingender, J., Szewzyk, U., Steinberg, P., Rice, S. A. y Kjelleberg, S. (2016). Biofilms: An emergent form of bacterial life. Nature Reviews Microbiology, 14 (9), pp. 563-575. https://doi. org/10.1038/nrmicro.2016.94

Gaglio, R., Cruciata, M., Gerlando, R. di, Scatassa, M. L., Cardamone, C., Mancuso, I., Sardina, M. T., Moschetti, G., Portolano, B. y Settanni, L. (2016). Microbial activation of wooden vats used for traditional cheese production and evolution of neoformed biofilms. Applied and Environmental Microbiology, 82 (2), pp. 585-595. https://doi. org/10.1128/AEM.02868-15

Gião, M. S. y Keevil, C. W. (2014). Listeria monocytogenes can form biofilms in tap water and enter into the viable but non-cultivable state. Microbial Ecology, 67 (3), pp. 603-611. https://doi. org/10.1007/s00248-013-0364-3

Giaouris, E., Chorianopoulos, N., Doulgeraki, A. y Nychas, G. J. (2013). Co-Culture with Listeria monocytogenes within a dual-species biofilm community strongly increases resistance of Pseudomonas putida to benzalkonium chloride. PLOS ONE, 8 (10), e77276. https://doi. org/10.1371/journal.pone.0077276

Giaouris, E., Heir, E., Desvaux, M., Hébraud, M., Møretrø, T., Langsrud, S., Doulgeraki, A., Nychas, G. J., Kačániová, M., Czaczyk, K., Ölmez, H. y Simões, M. (2015). Intra- and inter-species interactions within biofilms of important foodborne bacterial pathogens. Frontiers in Microbiology, 6, 841. https://doi. org/10.3389/fmicb.2015.00841

Gingichashvili, S., Duanis-Assaf, D., Shemesh, M., Featherstone, J. D. B., Feuerstein, O. y Steinberg, D. (2017). Bacillus subtilis biofilm development - a computerized study of morphology and kinetics. Frontiers in Microbiology, 8, 2072. https://doi.org/10.3389/ fmicb.2017.02072

Gkana, E. N., Doulgeraki, A. I., Chorianopoulos, N. G. y Nychas, G. J. E. (2017). Anti-adhesion and anti-biofilm potential of organosilane nanoparticles against foodborne pathogens. Frontiers in Microbiology, 8, 1295. https://doi. org/10.3389/fmicb.2017.01295 
Gomes, L. C., Deschamps, J., Briandet, R. y Mergulhão, F. J. (2018). Impact of modified diamond-like carbon coatings on the spatial organization and disinfection of mixed-biofilms composed of Escherichia coli and Pantoea agglomerans industrial isolates. International Journal of Food Microbiology, 277, pp. 74-82. https://doi. org/10.1016/j.ijfoodmicro.2018.04.017

González, S., Fernández, L., Campelo, A. B., Gutiérrez, D., Martínez, B., Rodríguez, A. y García, P. (2017). The behavior of Staphylococcus aureus dual-species biofilms treated with bacteriophage philPLA-RODI depends on the accompanying microorganism. Applied and Environmental Microbiology, 83 (3), e02821-16. https://doi.org/10.1128/AEM.02821-16

Gutiérrez, D., Rodríguez-Rubio, L., Martínez, B., Rodríguez, A. y García, P. (2016). Bacteriophages as weapons against bacterial biofilms in the food industry. Frontiers in Microbiology, 7, 825. https:// doi.org/10.3389/fmicb.2016.00825

Gutiérrez, D., Ruas-Madiedo, P., Martínez, B., Rodríguez, A. y García, P. (2014). Effective removal of Staphylococcal biofilms by the endolysin LysH5. PLOS ONE, 9 (9), e107307. https://doi. org/10.1371/journal.pone.0107307

Han, Q., Song, X., Zhang, Z., Fu, J., Wang, X., Malakar, P. K. Liu, H., Pan, Y. y Zhao, Y. (2017). Removal of foodborne pathogen biofilms by acidic electrolyzed water. Frontiers in Microbiology, 8, 988. https:// doi.org/10.3389/fmicb.2017.00988

Hayrapetyan, H., Muller, L., Tempelaars, M., Abee, T. y Nierop Groot, M. (2015). Comparative analysis of biofilm formation by Bacillus cereus reference strains and undomesticated food isolates and the effect of free iron. International Journal of Food Microbiology, 200, pp. 72-79. https://doi.org/10.1016/j.ijfoodmicro.2015.02.005

Heir, E., Møretrø, T., Simensen, A. y Langsrud, S. (2018). Listeria monocytogenes strains show large variations in competitive growth in mixed culture biofilms and suspensions with bacteria from food processing environments. International Journal of Food Microbiology, 275, pp. 46-55. https://doi. org/10.1016/j.ijfoodmicro.2018.03.026

Herschend, J., Damholt, Z. B. V., Marquard, A. M., Svensson, B., Sørensen, S. J., Hägglund, P. y Burmølle, M. (2017). A metaproteomics approach to study the interspecies interactions affecting microbial biofilm development in a model community. Scientific Reports, 7 (1), 16483. https://doi.org/10.1038/s41598-01716633-6

Hsu, L. C., Fang, J., Borca-Tasciuc, D. A., Worobo, R. W. y Moraru, C. I. (2013). Effect of micro- and nanoscale topography on the adhesion of bacterial cells to solid surfaces. Applied and Environmental Microbiology, 79 (8), pp. 2703-2712. https://doi.org/10.1128/AEM.03436-12

Huang, K., Chen, J., Nugen, S. R. y Goddard, J. M. (2016). Hybrid antifouling and antimicrobial coatings prepared by electroless co-deposition of fluoropolymer and cationic silica nanoparticles on stainless steel: efficacy against Listeria monocytogenes. ACS Applied Materials and Interfaces, 8 (25), pp. 15926-15936. https://doi.org/10.1021/ acsami.6b04187

Hussain, M. S., Kwon, M., Tango, C. N. y Oh, D. H. (2018). Effect of electrolyzed water on the disinfection of Bacillus cereus biofilms: the mechanism of enhanced resistance of sessile cells in the biofilm matrix. Journal of Food Protection, 81 (5), pp. 860-869. https://doi. org/10.4315/0362-028X.JFP-17-450

Hüwe, C., Schmeichel, J., Brodkorb, F., Dohlen, S., Kalbfleisch, K., Kreyenschmidt, M., Lorenz, R. y Kreyenschmidt, J. (2018). Potential of antimicrobial treatment of linear low-density polyethylene with poly((tert-butylamino)-methyl-styrene) to reduce biofilm formation in the food industry. Biofouling, 34 (4), pp. 378-387. https://doi. org/10.1080/08927014.2018.1453926

Iliadis, I., Daskalopoulou, A., Simões, M. y Giaouris, E. (2018). Integrated combined effects of temperature, $\mathrm{pH}$ and sodium chloride concentration on biofilm formation by Salmonella enterica ser. Enteritidis and Typhimurium under low nutrient food-related conditions. Food Research International, 107 pp. 10-18. https://doi.org/10.1016/j. foodres.2018.02.015

Jahid, I. K., Lee, N.-Y., Kim, A. y Ha, S.-D. (2013). Influence of glucose concentrations on biofilm formation, motility, exoprotease production, and quorum sensing in Aeromonas hydrophila. Journal of Food Protection, 76 (2), pp. 239 247. https://doi.org/10.4315/0362028X.JFP-12-321

Jeon, H. R., Kwon, M. J. y Yoon, K. S. (2018). Control of Listeria innocua biofilms on food contact surfaces with slightly acidic electrolyzed water and the risk of biofilm cells transfer to duck meat. Journal of Food Protection, 81 (4), pp. 582-592. https://doi.org/10.4315/0362-028X. JFP-17-373

Jindal, S., Anand, S., Metzger, L. y Amamcharla, J. (2018). Short communication: A comparison of biofilm development on stainless steel and modified-surface plate heat exchangers during a 17-h milk pasteurization run. Journal of Dairy Science, 101 (4), pp. 2921-2926. https:// doi.org/10.3168/jds.2017-14028

Kadam, S. R., den Besten, H. M. W., van der Veen, S., Zwietering, M. H., Moezelaar, R. y Abee, T. (2013). Diversity assessment of Listeria monocytogenes biofilm formation: Impact of growth condition, serotype and strain origin. International Journal of Food Microbiology, 165 (3), pp. 259-264. https://doi.org/10.1016/j. ijfoodmicro.2013.05.025

Kim, S., Bang, J., Kim, H., Beuchat, L. R. y Ryu, J. H. (2013). Inactivation of Escherichia coli 0157: $\mathrm{H} 7$ on stainless steel upon exposure to Paenibacillus polymyxa biofilms. International Journal of Food Microbiology, 167 (3), pp. 328336. https://doi.org/10.1016/j.ijfoodmicro.2013.10.004

Kim, M. K., Zhao, A., Wang, A., Brown, Z. Z., Muir, T. W., Stone, H. A. y Bassler, B. L. (2017). Surface-attached molecules control Staphylococcus aureus quorum sensing and biofilm development. $\mathrm{Na}$ ture Microbiology, 2 (8), 17080. https:// doi.org/10.1038/nmicrobiol.2017.80

Kiran, G. S., Lipton, A. N., Kennedy, J., Dobson, A. D. W. y Selvin, J. (2014). A halotolerant thermostable lipase from the marine bacterium Oceanobacillus sp. PUMB02 with an ability to disrupt bacterial biofilms. Bioengineered Bugs, 5 (5), pp. 305-318. https://doi.org/10.4161/bioe.29898

Larsen, M. H., Dalmasso, M., Ingmer, H., Langsrud, S., Malakauskas, M., Mader, A., Møretrø, T., Možina, S. S., Rychli, K., Wagner, R., Wallace, R. J., Zentek, J. y Jordan, K. (2014). Persistence of foodborne pathogens and their control in primary and secondary food production chains. Food Control, 44, pp. 92-109. https://doi. org/10.1016/j.foodcont.2014.03.039

Li, J., Feng, J., Ma, L., Fuente Núñez, C. de la, Gölz, G. y Lu, X. (2017). Effects of meat juice on biofilm formation of Campylobacter and Salmonella. International Journal of Food Microbiology, 253, pp. 
20-28. https://doi.org/10.1016/j.ijfoodmicro.2017.04.013

Liu, J., Prindle, A., Humphries, J., GabaldaSagarra, M., Asally, M., Lee, D. Y. D., Ly, S. y Süel, G. M. (2015). Metabolic co-dependence gives rise to collective oscillations within biofilms. Nature, 523 (7562), pp. 550-554. https://doi.org/10.1038/ nature14660

Mai-Prochnow, A., Clauson, M., Hong, J. y Murphy, A. B. (2016). Gram positive and Gram negative bacteria differ in their sensitivity to cold plasma. Scientific Reports, 6 (1), 38610. https://doi. org/10.1038/srep38610

Makovcova, J., Babak, V., Kulich, P., Masek, J., Slany, M. y Cincarova, L. (2017). Dynamics of mono- and dual-species biofilm formation and interactions between Staphylococcus aureus and Gram-negative bacteria. Microbial Biotechnology, 10 (4), pp. 819-832. https:// doi.org/10.1111/1751-7915.12705

Mariani, C., Oulahal, N., Chamba, J. F., Dubois-Brissonnet, F., Notz, E. y Briandet, R. (2011). Inhibition of Listeria monocytogenes by resident biofilms present on wooden shelves used for cheese ripening. Food Control, 22 (8), pp. 13571362. https://doi.org/10.1016/j.foodcont.2011.02.012

Marti, R., Schmid, M., Kulli, S., Schneeberger, K., Naskova, J., Knøchel, S. Ahrens, C. H. y Hummerjohann, J. (2017). Biofilm formation potential of heat-resistant Escherichia coli dairy isolates and the complete genome of multidrug-resistant, heat-resistant strain FAM21845. Applied and Environmental Microbiology, 83 (15), e00628-17. https://doi. org/10.1128/AEM.00628-17

Martin, J. G. P., Oliveira e Silva, G. de, Fonseca, C. R. da, Morales, C. B., Souza Pamplona Silva, C., Miquelluti, D. L. y Porto, E. (2016). Efficiency of a cleaning protocol for the removal of enterotoxigenic Staphylococcus aureus strains in dairy plants. International Journal of Food Microbiology, 238, pp. 295-301. https://doi.org/10.1016/j.ijfoodmicro.2016.09.018

McKenzie, K., Maclean, M., Timoshkin, I. V., Endarko, E., Macgregor, S. J. y Anderson, J. G. (2013). Photoinactivation of bacteria attached to glass and acrylic surfaces by $405 \mathrm{~nm}$ light: Potential application for biofilm decontamination. Photochemistry and Photobiology, 89 (4), pp. $927-$ 935. https://doi.org/10.1111/php.12077
Montgomery, N. L. y Banerjee, P. (2015). Inactivation of Escherichia coli 0157:H7 and Listeria monocytogenes in biofilms by pulsed ultraviolet light. BMC Research Notes, 8 (1), 235. https://doi. org/10.1186/s13104-015-1206-9

Moradi, M. y Tajik, H. (2017). Biofilm removal potential of neutral electrolysed water on pathogen and spoilage bacteria in dairy model systems. Journal of Applied Microbiology, 123 (6), pp. 1429-1437. https://doi.org/10.1111/ jam. 13608

Nadell, C. D., Drescher, K. y Foster, K. R. (2016). Spatial structure, cooperation and competition in biofilms. Nature Reviews Microbiology, 14 (9), pp. 589600. https://doi.org/10.1038/nrmicro.2016.84

Nam, H., Seo, H. S., Bang, J., Kim, H., Beuchat, L. R. y Ryu, J. H. (2014). Efficacy of gaseous chlorine dioxide in inactivating Bacillus cereus spores attached to and in a biofilm on stainless steel. International Journal of Food Microbiology, 188, pp. 122-127. https://doi. org/10.1016/j.ijfoodmicro.2014.07.009

Nguyen, U. T. y Burrows, L. L. (2014). DNase $\mathrm{I}$ and proteinase $\mathrm{K}$ impair Listeria monocytogenes biofilm formation and induce dispersal of pre-existing biofilms. International Journal of Food Microbiology, 187, pp. 26-32. https://doi. org/10.1016/j.ijfoodmicro.2014.06.025

Nicholas, R., Dunton, P., Tatham, A. y Fielding, L. (2013). The effect of ozone and open air factor on surface-attached and biofilm environmental Listeria monocytogenes. Journal of Applied Microbiology, 15 (2), pp. 555-564. https://doi. org/10.1111/jam.12239

Niemira, B. A., Boyd, G. y Sites, J. (2014). Cold plasma rapid decontamination of food contact surfaces contaminated with Salmonella biofilms. Journal of Food Science, 79 (5), M917-M922. https://doi.org/10.1111/17503841.12379

Nowak, J., Cruz, C. D., Tempelaars, M., Abee, T., van Vliet, A. H. M., Fletcher, G. C., Hedderley, D., Palmer, J. y Flint, S. (2017). Persistent Listeria monocytogenes strains isolated from mussel production facilities form more biofilm but are not linked to specific genetic markers. International Journal of Food Microbiology, 256, pp. 45-53. https://doi. org/10.1016/j.ijfoodmicro.2017.05.024
Ortiz, S., López, V. y Martínez-Suárez, J. V. (2014). The influence of subminimal inhibitory concentrations of benzalkonium chloride on biofilm formation by Listeria monocytogenes. International Journal of Food Microbiology, 189, pp. 106-112. https://doi.org/10.1016/j.ijfoodmicro.2014.08.007

Overney, A., Jacques-André-Coquin, J., Ng, P., Carpentier, B., Guillier, L. y Firmesse, O. (2017). Impact of environmental factors on the culturability and viability of Listeria monocytogenes under conditions encountered in food processing plants. International Journal of Food Microbiology, 244, pp. 74-81. https://doi. org/10.1016/j.ijfoodmicro.2016.12.012

Papaioannou, E., Giaouris, E. D., Berillis, P. y Boziaris, I. S. (2018). Dynamics of biofilm formation by Listeria monocytogenes on stainless steel under monospecies and mixed-culture simulated fish processing conditions and chemical disinfection challenges. International Journal of Food Microbiology, 267, pp. 9-19. https://doi.org/10.1016/j.ijfoodmicro.2017.12.020

Pasvolsky, R., Zakin, V., Ostrova, I. y Shemesh, M. (2014). Butyric acid released during milk lipolysis triggers biofilm formation of Bacillus species. International Journal of Food Microbiology, 181, pp. 19-27. https://doi.org/10.1016/j.ijfoodmicro.2014.04.013

Puligundla, P. y Mok, C. (2017). Potential applications of nonthermal plasmas against biofilm-associated micro-organisms in vitro. Journal of Applied Microbiology, 122 (5), pp. 1134-1148. https:// doi.org/10.1111/jam.13404

Røder, H. L., Raghupathi, P. K., Herschend, J., Brejnrod, A., Knøchel, S., Sørensen, S. J. y Burmølle, M. (2015). Interspecies interactions result in enhanced biofilm formation by co-cultures of bacteria isolated from a food processing environment. Food Microbiology, 51, pp. 18-24. https://doi.org/10.1016/j. fm.2015.04.008

Rodríguez-López, P., Saá-Ibusquiza, P., Mosquera-Fernández, M. y López-Cabo, M. (2015). Listeria monocytogenes-carrying consortia in food industry. Composition, subtyping and numerical characterisation of mono-species biofilm dynamics on stainless steel. International Journal of Food Microbiology, 206, pp. 84-95. https://doi.org/10.1016/j.ijfoodmicro.2015.05.003 
Rosenberg, G., Steinberg, N., OppenheimerShaanan, Y., Olender, T., Doron, S., BenAri, J., Sirota-Madi, A, Bloom-Ackermann, Z. y Kolodkin-Gal, I. (2016). Not so simple, not so subtle: The interspecies competition between Bacillus simplex and Bacillus subtilis and its impact on the evolution of biofilms. NPJ Biofilms and Microbiomes, 2 (1), 15027. https://doi. org/10.1038/npjbiofilms.2015.27

Sadekuzzaman, M., Yang, S., Mizan, M. F. R. y Ha, S. D. (2017). Reduction of Escherichia coli 0157:H7 in biofilms using bacteriophage BPECO 19. Journal of Food Science, 82 (6), pp. 1433-1442. https://doi. org/10.1111/1750-3841.13729

Sepehr, S., Rahmani-Badi, A., Babaie-Naiej, H. y Soudi, M. R. (2014). Unsaturated fatty acid, cis-2-decenoic acid, in combination with disinfectants or antibiotics removes pre-established biofilms formed by food-related bacteria. PLoS ONE, 9 (7), e101677. https://doi. org/10.1371/journal.pone.0101677

Shafique, M., Alvi, I. A., Abbas, Z. y ur Rehman, S. (2017). Assessment of biofilm removal capacity of a broad host range bacteriophage JHP against Pseudomonas aeruginosa. APMIS, 125 (6) pp. 579-584. https://doi.org/10.1111/ apm.12691

Silva Fernandes, M. da, Kabuki, D. Y. y Kuaye, A. Y. (2015). Behavior of Listeria monocytogenes in a multi-species biofilm with Enterococcus faecalis and Enterococcus faecium and control through sanitation procedures. International Journal of Food Microbiology, 200, pp. 5-12. https://doi.org/10.1016/j.ijfoodmicro.2015.01.003

Skovager, A., Larsen, M. H., Castro-Mejia, J. L., Hecker, M., Albrecht, D., Gerth, U., Arneborg, N. y Ingmer, H. (2013). Initial adhesion of Listeria monocytogenes to fine polished stainless steel under flow conditions is determined by prior growth conditions. International Journal of Food Microbiology, 165 (1), pp. 35-42. https://doi.org/10.1016/j.ijfoodmicro.2013.04.014

Slany, M., Oppelt, J. y Cincarova, L. (2017). Formation of Staphylococcus aureus biofilm in the presence of sublethal concentrations of disinfectants studied via a transcriptomic analysis using transcriptome sequencing (RNA-seq). Applied and Environmental Microbiology, 83 (24), e01643-17. https://doi. org/10.1128/AEM.01643-17
Son, H., Park, S., Beuchat, L. R., Kim, H. y Ryu, J. H. (2016). Inhibition of Staphylococcus aureus by antimicrobial biofilms formed by competitive exclusion microorganisms on stainless steel. International Journal of Food Microbiology, 238, pp. 165-171. https://doi. org/10.1016/j.ijfoodmicro.2016.09.007

Stevens, M. R. E., Luo, T. L., Vornhagen, J., Jakubovics, N. S., Gilsdorf, J. R., Marrs, C. F., Møretrø, T. y Rickard, A. H. (2015). Coaggregation occurs between microorganisms isolated from different environments. FEMS Microbiology Ecology, 91 (11), fiv123. https://doi.org/10.1093/ femsec/fiv123

Tack, I. L. M. M., Nimmegeers, P., Akkermans, S., Hashem, I. y van Impe, J. F. M. (2017). Simulation of Escherichia coli dynamics in biofilms and submerged colonies with an individual-based model including metabolic network information. Frontiers in Microbiology, 8, 2509. https://doi.org/10.3389/ fmicb.2017.02509

Tarifa, M. C., Genovese, D., Lozano, J. E. y Brugnoni, L. I. (2018). In situ microstructure and rheological behavior of yeast biofilms from the juicprocessing industries. Biofouling, 34 (1), pp. 74-85. https://doi.org/10.1080/08927014.201 7.1407758

Techaruvichit, P., Takahashi, H., Kuda, T., Miya, S., Keeratipibul, S. y Kimura, B. (2016). Adaptation of Campylobacter jejuni to biocides used in the food industry affects biofilm structure, adhesion strength, and cross-resistance to clinical antimicrobial compounds. Biofouling, 32 (7), pp. 827-839. https://doi. org/10.1080/08927014.2016.1198476

Turonova, H., Briandet, R., Rodrigues, R., Hernould, M., Hayek, N., Stintzi, A., Pazlarova, J. y Tresse, O. (2015). Biofilm spatial organization by the emerging pathogen Campylobacter jejuni: Comparison between NCTC 11168 and 81-176 strains under microaerobic and oxygen-enriched conditions. Frontiers in Microbiology, 6, 709. https://doi. org/10.3389/fmicb.2015.00709

Visvalingam, J., Ells, T. C. y Yang, X. (2017). Impact of persistent and nonpersistent generic Escherichia coli and Salmonella sp. recovered from a beef packing plant on biofilm formation by $E$. coli O157. Journal of Applied Microbiology, 123 (6), pp. 1512-1521. https://doi. org/10.1111/jam.13591
Vogeleer, P., Tremblay, Y. D. N., Jubelin, G., Jacques, M. y Harel, J. (2016). Biofilmforming abilities of Shiga toxin-producing Escherichia coli isolates associated with human infections. Applied and Environmental Microbiology, 82 (5), pp. 1448-1458. https://doi.org/10.1128/ AEM.02983-15

Wang, R., Kalchayanand, N., Schmidt, J. W. y Harhay, D. M. (2013). Mixed biofilm formation by Shiga Toxin-Producing Escherichia coli and Salmonella enterica Serovar Typhimurium enhanced bacterial resistance to sanitization due to extracellular polymeric substances. Journal of Food Protection, 76 (9), pp. 15131522. https://doi.org/10.4315/0362028X.JFP-13-077

Wang, J., Ray, A. J., Hammons, S. R. y Oliver, H. F. (2015). Persistent and transient Listeria monocytogenes strains from retail deli environments vary in their ability to adhere and form biofilms and rarely have inlA premature stop codons. Foodborne Pathogens and Disease, 12 (2), pp. 151-158. https://doi.org/10.1089/ fpd.2014.1837

Xue, T., Chen, X. y Shang, F. (2014). Short communication: Effects of lactose and milk on the expression of biofilm-associated genes in Staphylococcus aureus strains isolated from a dairy cow with mastitis. Journal of Dairy Science, 97 (10), pp. 6129-6134. https://doi. org/10.3168/jds.2014-8344

Yu, S., Su, T., Wu, H., Liu, S., Wang, D., Zhao, T., Jin, Z., Du, W., Zhu, M.-J., Chua, S. L., Yang, L., Zhu, D., Gu, L. y Ma, L. Z. (2015). PsIG, a self-produced glycosyl hydrolase, triggers biofilm disassembly by disrupting exopolysaccharide matrix. Cell Research, 25 (12), pp. 1352-1367. https://doi.org/10.1038/cr.2015.129

Zhao, T., Podtburg, T. C., Zhao, P., Chen, D., Baker, D. A., Cords, B. y Doyle, M. P. (2013). Reduction by competitive bacteria of Listeria monocytogenes in biofilms and Listeria bacteria in floor drains in a ready-to-eat poultry processing plant. Journal of Food Protection, 76 (4), pp. 601-607. https://doi. org/10.4315/0362-028X.JFP-12-323

Ziuzina, D., Boehm, D., Patil, S., Cullen, P. J. y Bourke, P. (2015). Cold plasma inactivation of bacterial biofilms and reduction of quorum sensing regulated virulence factors. PLOS ONE, 10 (9), e0138209. https://doi.org/10.1371/journal. pone.0138209 